\title{
Case Report: A New Mycobacterium ulcerans Genotype Causing Buruli Ulcer in Côte d'Ivoire
}

\author{
Philippe Bahadoran, ${ }^{1}$ Nassim Hammoudi, ${ }^{2,3}$ Alice Gaudart, ${ }^{4}$ Jamal Saad, ${ }^{2,3}$ Yoan Di Filippo, ${ }^{1}$ Michel Drancourt, ${ }^{2,3}$ and \\ Raymond Ruimy ${ }^{4,5,6 *}$
}

${ }^{1}$ Department of Dermatology, Centre Hospitalier Universitaire de Nice, Université Côte D'Azur, Inserm, Nice, France; ${ }^{2} I R D, M E P H I$, IHU Méditerranée Infection, Aix-Marseille-Université, Marseille, France; ${ }^{3} I H U$ Méditerranée Infection, Marseille, France; ${ }^{4}$ Laboratoire de Bactériologie, Centre Hospitalier Universitaire de Nice, Hôpital de l'Archet II, Nice, France; ${ }^{5}$ Université Côte D'Azur, Inserm, C3M, Nice, France; ' Inserm U1065, C3M, Equipe 6, Nice, France

\begin{abstract}
Mycobacterium ulcerans, the opportunistic pathogen causing Buruli ulcer, is reported to affect rural populations in 36 tropical countries. We report one case of Buruli ulcer in a peri-urban area in Côte d'Ivoire, confirmed by whole genome sequencing which indicated a M. ulcerans genotype previously unreported in Côte d'Ivoire.
\end{abstract}

Buruli ulcer is a debilitating, chronic infection of the skin and the subcutaneous tissues caused by the nontuberculous Mycobacterium ulcerans, first described 80 years ago in Australia. ${ }^{1}$ Mycobacterium ulcerans is an opportunistic pathogen that exists in poorly defined swampy environments in 36 notifying countries largely located in tropical regions, with West African countries, including Côte d'Ivoire, reporting the largest number of cases to the $\mathrm{WHO} .^{2,3}$ In these African countries, Buruli ulcer affects rural populations exposed to environments contaminated with $M$. ulcerans. ${ }^{3}$ Mycobacterium ulcerans secretes proapoptotic exotoxin mycolactones which are the main virulence factors of the pathogen, promoting tissue necrosis, an anti-inflammatory response, and analgesia. $^{4}$

A 56-year-old man consulted for a 6-month evolving ulcer on the front of his left leg, which he reported as an "ingrown hair," while living in the vicinity of Abidjan in Côte d'Ivoire, for 23 years. The patient had a 15-year history of mild Behçet disease (aphthosis and arthralgia), which was treated with colchicine. The patient denied walking barefoot and having any contact with stagnant water such as lakes or backwaters while being in Côte d'Ivoire. He had not lived in any other African countries or in any other Buruli ulcer-endemic country. The patient confirmed that he had always lived in the city of Grand-Bassam, located $35 \mathrm{~km}$ east of downtown Abidjan. At the time of the first consultation, the patient was apyretic and the clinical examination was unremarkable, except for a $2.5-\mathrm{cm}$ violaceous inflammatory nodule surrounding a yellowish fibrinous ulceration. The skin beyond the lesion manifested a tendency to peel away from the adjacent tissues (Figure 1A). Histopathologic examination of a lesion biopsy showed epidermal hyperplasia with a nonspecific inflammatory infiltrate. Microscopic examination of the biopsy lesion showed no microorganism after periodic acid Schiff, Grocott, and Gram stainings and no acid-fast bacilli following Kinyoun and auramine stainings. However, colonies observed on the Coletsos medium after incubating the biopsy lesion for 42 days at $30^{\circ} \mathrm{C}$ were identified as M. ulcerans by the GenoType Mycobacterium CM/AS assay (Biocentric, Bandol, France). Total DNA extracted from this M. ulcerans CSURQ0185 isolate using the InstaGene matrix (Bio-Rad, Marnes-la-Coquette, France) was sequenced

*Address correspondence to Raymond Ruimy, Laboratoire de Bacteriologie, 151, Route de St Antoine de Ginestière, Nice 06000, France. E-mail: ruimy.r@chu-nice.fr using a MiSeq platform (Illumina, Inc., San Diego, CA). Plasmid detection was performed using Megablast (Optimize for highly similar sequences) against the Nucleotide collection database (nr/nt). Pangenome study including CSURQ0185 strain genome, 327 other $M$. ulcerans genomes (including 20/327 genomes from Cote d'Ivoire isolates), three Mycobacterium pseudoshottsii, three Mycobacterium liflandii, and $24 M$. marinum genomes (Supplemental Table S1) yielded 2,804 core genes $(99 \% \leq$ strains $\leq 100 \%), 783$ soft core genes $(95 \% \leq$ strains < $99 \%), 2,155$ shell genes $(15 \% \leq$ strains $<95 \%$ ), and 48,469 cloud genes ( $0 \% \leq$ strains $<15 \%$ ). The core genome phylogenetic tree (Figure 2) and single nucleotide polymorphism (SNP) distance showed that

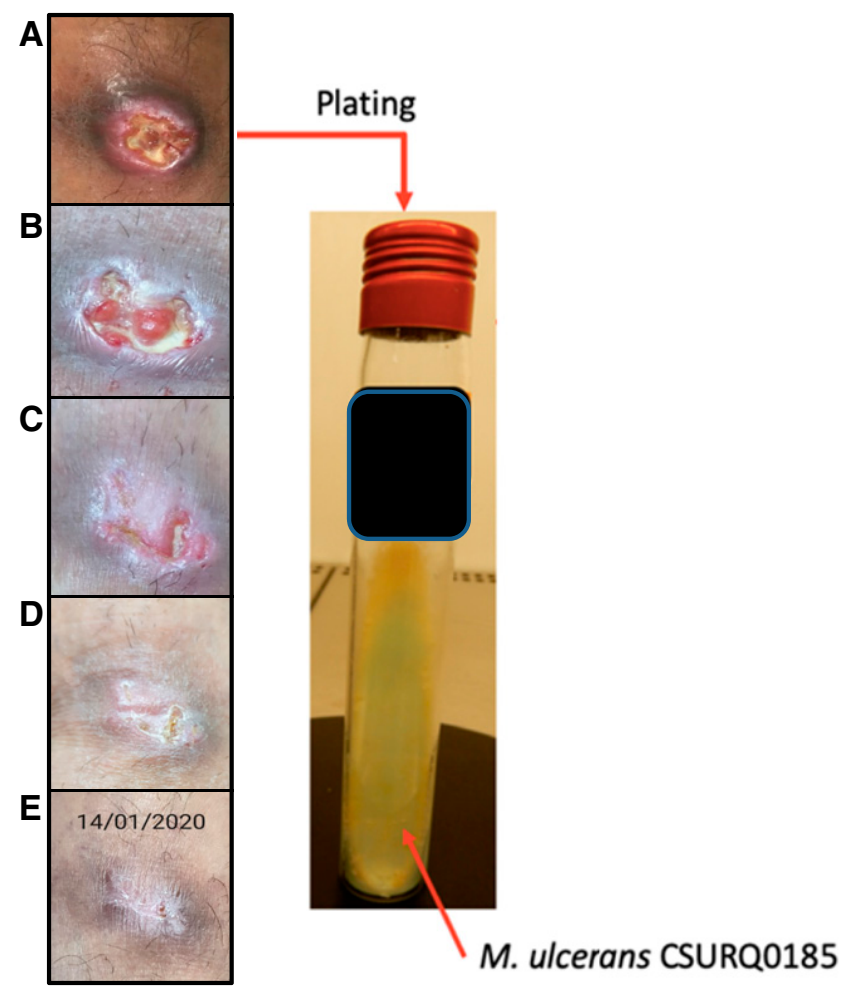

FIGURE 1. Investigation of one case of peri-urban Buruli ulcer, Côte d'Ivoire: chronological evolution of the lesion under treatment (from top to down). Images of (A) first consultation, (B) initiation of treatment, (C) 2-month treatment, (D) 4-month treatment, and (E) 6-month treatment; middle panel features Mycobacterium ulcerans colonies on Coletsos culture medium. This figure appears in color at www.ajtmh.org. 


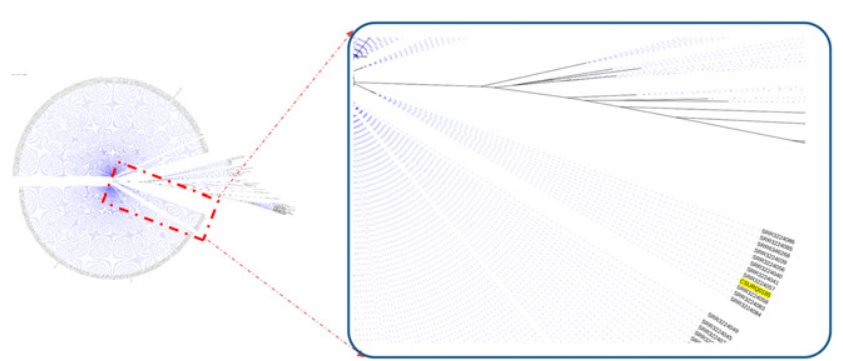

FiguRE 2. Phylogenetic tree based on extracted core genome of 385 Mycobacterium ulcerans genomes using Roary, ${ }^{11}$ inferred with MAFFT version 7 and reconstructed using iTOL (https://itol.embl.de/ upload.cgi). This figure appears in color at www.ajtmh.org.

CSURQ0185 strain was clustered with other M. ulcerans strains including SRR3224058 (14-SNP; Benin), SRR3224057 (15-SNP; Benin), SRR3224084 (17-SNP; Benin), SRR3224083 (24-SNP; Benin), SRR3224039 (54-SNP; Cameroon), SRR3224041 (61SNP; Gabon), SRR3224056 (69-SNP; Benin), SRR3224040 (72SNP; Gabon), SRR3224086 (346-SNP; Papua New Guinea), SRR3224085 (349-SNP; Papua New Guinea), and SRR6346268 (351-SNP; Papua New Guinea) (Supplemental Table S1). Accordingly, this $11 \mathrm{M}$. ulcerans strain cluster, including CSURQ0185 strain, exhibited SNP distance $>2,000$ with the other M. ulcerans strains, including Côte d'lvoire strains (Supplemental Table S2). Further comparison with the Comprehensive Antibiotic Resistance Database ${ }^{5}$ predicted M. ulcerans CSURQ0185 to be resistant to aminoglycosides, monobactam, cephalosporin, fosfomycin, and paraaminosalicylic acid. As for macrolides, A2274G mutation in 23S rRNA gene confers resistance in Mycobacterium avium only, and we detected erm gene with $66.7 \%$ protein identity. Meanwhile, in the absence of any specific treatment, inflammatory borders of the lesion disappeared, and the ulceration had grown insidiously (Figure 1B). Once the identification of M. ulcerans had been confirmed by whole genome sequencing and the diagnosis of Buruli ulcer firmly established, reference treatment with rifampicin $10 \mathrm{mg} / \mathrm{kg} /$ day and azithromycin 15 $\mathrm{mg} / \mathrm{kg} /$ day was started, and colchicine was stopped to avoid any lethal cardiac side effects of the azithromycin and colchicine association. The patient continued the antibiotic treatment for 6 months, resulting in a progressive and nearcomplete healing of the ulcer (Figure 1A-E).

Regarding this patient, Buruli ulcer was caused by a M. ulcerans strain, the genome sequence of which was distant from Côte d'Ivoire isolates and more closely related to M. ulcerans isolates from Benin, Cameroon, Gabon, and Papua New Guinea. These unprecedented results suggest the recent introduction of the $M$. ulcerans genotype in Côte d'Ivoire, where it had never been previously detected. The fact that the same $M$. ulcerans genotype was detected in several, noncontiguous West African countries has been previously reported, questioning the routes of migration of an otherwise nonmotile pathogen. ${ }^{6}$ However, these data must be balanced by the fact that the actual genomic diversity of $M$. ulcerans in the environment is unknown, in the absence of any environmental isolate (except one case from one Gerris $\mathrm{sp}$. in Ghana) ${ }^{7}$ and any whole genome sequence data issued from metagenomic data from environmental niches where $M$. ulcerans is thriving. Interestingly, this patient denied any exposure to Buruli ulcer-endemic regions in Côte d'Ivoire, as he remained in the vicinity of the city of Abidjan where Buruli ulcer cases have not yet been confirmed.

This case illustrates a shift in the epidemiology of Buruli ulcer in Côte d'Ivoire, from an exclusively rural infection toward a peri-urban one. The same epidemiological shift is probably one of several factors supporting the reemergence of Buruli ulcer in southern Australia, ${ }^{8}$ illustrating that Buruli ulcer is a prototype opportunist pathogen entirely dependent on the ecosystem, including populations and their contacts with rural areas.

Received October 6, 2020. Accepted for publication January 18, 2021. Published online April 5, 2021.

Note: Supplemental tables appear at www.ajtmh.org.

Financial support: N. H. obtained a PhD grant from the Fondation Méditerranée Infection, Marseille, France. This work was supported by the Agence Nationale de la Recherche (ANR-17-CE35-0006-01 PRIME, http://www.agencenationale-recherche.fr/) and by the French government under the "Investissements d'Avenir" (Investments in the Future) program managed by the A. N. R. (reference: Méditerranée Infection 10-IAHU-03). This work was supported by Région Le Sud (Provence Alpes Côte d'Azur) and European FEDER IHUBIOTK funding.

Authors' addresses: Philippe Bahadoran and Yoan Di Filippo, Centre Hospitalier Universitaire de Nice, Dermatology, Nice, ProvenceAlpes-Côte d'Azur, France, E-mails: bahadoran.P@chu-nice.fr and difilippo.y@chu-nice.fr. Nassim Hammoudi, Jamal Saad, and Michel Drancourt, Aix-Marseille Universite, MEPHI, Marseille, ProvenceAlpes-Côte d'Azu, France, E-mails: nassimveto15@live.fr, jsaad270@ gmail.com, and michel.drancourt@univ-amu.fr. Alice Gaudart, Laboratoire de Bactériologie, Centre Hospitalier, Universitaire de Nice, Hôpital de I'Archet II, Nice, France, E-mail: gaudart.a@chu-nice.fr. Raymond Ruimy, Laboratoire de Bactériologie, Centre Hospitalier Universitaire de Nice, Hôpital de l'Archet II, Laboratoire de Bacteriologie, 151, Route de St Antoine de Ginestière, Nice, France, and Inserm, C3M, Team 6, Nice, France, E-mail: ruimy.r@chu-nice.fr.

\section{REFERENCES}

1. MacCallum P, Tolhurst JC, Buckle G, Sissons HA, 1948. A new mycobacterial infection in man. J Pathol Bacteriol 60: 93-102.

2. Aubry P, Gaüzère BA, 2020. Ulcère de Buruli. Available at: http:// medecinetropicale.free.fr/cours/ulcere_buruli.pdf. Accessed Januray 22, 2020.

3. Zingue D, Bouam A, Tian RBD, Drancourt M, 2017. Buruli ulcer, a prototype for ecosystem-related infection, caused by Mycobacterium ulcerans. Clin Microbiol Rev 31: e00045-17.

4. George KM et al., 1999 Mycolactone: a polyketide toxin from Mycobacterium ulcerans required for virulence. Science 283: 854-857.

5. Alcock BP et al., 2020. Card 2020: antibiotic resistome surveillance with the comprehensive antibiotic resistance database. Nucleic Acids Res 48: D517-D525.

6. Buultjens $\mathrm{AH}$ et al., 2018. Comparative genomics shows that Mycobacterium ulcerans migration and expansion preceded the rise of Buruli ulcer in southeastern Australia. Appl Environ Microbiol 84: e02612-17.

7. Portaels $\mathrm{F}$ et al., 2008. First cultivation and characterization of Mycobacterium ulcerans from the environment. PLoS Negl Trop Dis 2: e178.

8. Tai AYC, Athan E, Friedman ND, Hughes A, Walton A, O'Brien DP, 2018. Increased severity and spread of Mycobacterium ulcerans, southeastern Australia. Emerg Infect Dis 24: 58-64.

9. Vandelannoote $\mathrm{K}$ et al., 2017. Multiple introductions and recent spread of the emerging human pathogen Mycobacterium ulcerans across Africa. Genome Biol Evol 9: 414-426.

10. Hammoudi N, Saad J, Drancourt M, 2020. The diversity of mycolactone-producing mycobacteria. Microb Pathog 149: 104362.

11. Page AJ et al., 2015. Roary: rapid large-scale prokaryote pan genome analysis. Bioinformatics 31: 3691-3693. 